\title{
Determinación del volumen tiroideo por ecografía en población adulta asintomática de la ciudad de Pereira - Colombia
}

\author{
Determination of thyroid volume by ultrasound in adult population \\ without thyroid pathology from Pereira - Colombia
}

\author{
Arenas HM1, López $V^{1}$, Arias DR ${ }^{2}$, Orozco-Hernández JP $P^{3}$,Estrada-Álvarez JM ${ }^{4}$
}

\begin{abstract}
${ }^{1}$ Endocrinólogo, Clínica Comfamiliar; profesor de Medicina Interna, Universidad Tecnológica de Pereira, Colombia.

${ }^{2}$ Residente de Medicina Interna, Universidad Tecnológica de Pereira, Pereira, Colombia.

${ }^{3}$ Médico e Investigador, Grupo de Investigación Salud Comfamiliar, Pereira; Programa de Medicina, Universidad Tecnológica de Pereira, Colombia.

${ }^{4}$ Magister en Estadística aplicada, candidato a Doctor en Estadística Matemática y Aplicada, Grupo de Investigación Salud Comfamiliar, Pereira, Colombia.
\end{abstract}

Autor de correspondencia: Henry Mauricio Arenas

Correo electrónico: hmaq@hotmail.com

Fecha de recepción: 14/09/2019

Fecha de aceptación: 10/12/2019

\section{Resumen}

Introducción: el aumento del volumen tiroideo puede presentarse tanto en hipertiroidismo como en hipotiroidismo. Los valores de referencia en la población colombiana de acuerdo con grupos etarios no se han establecido, lo que obliga a usar fuentes de otra población de características diferentes. Lo anterior genera errores en la práctica clínica diaria y conlleva a conductas equívocas, por lo que es importante obtener valores de referencia normales del tamaño tiroideo en la población. El objetivo de este estudio fue estimar los valores de referencia para el volumen tiroideo normal en la población adulta de la ciudad de Pereira sin anomalía tiroidea.

Métodos: estudio observacional de corte trasversal realizado en la Clínica Comfamiliar Risaralda, con población masculina y femenina mayor de 18 años sin patología tiroidea previa. Se estimaron las ecuaciones de regresión en polinomios fraccionales para los valores medios y su correspondiente variabilidad en el volumen tiroideo en función de la edad; se obtuvieron los centiles por edad. Resultados: se incluyó un total de 170 pacientes al estudio. El volumen total promedio de la glándula tiroides en la población fue de 6,5 $\pm 2,1 \mathrm{~mL}$, siendo mayor en el sexo masculino (media 7,9 $\pm 1,7 \mathrm{~mL}$ ) que en el femenino (media: 6,0 $\pm 2,0 \mathrm{~mL}$ ). Se identificó una tendencia estable del volumen tiroideo hasta los 51 años (p50: 6,54 mL) con una posterior tendencia al incremento hasta los 69 años (p50: 9,64 mL).

Conclusiones: se obtuvieron valores de referencia normales de volumen tiroideo en la población pereirana adulta sana.

Palabras clave: glándula tiroides, ultrasonografía, Colombia.

\section{Abstract}

Introduction: abnormalities in thyroid volume can occur in hyperthyroidism and in hypothyroidism. Reference values for thyroid volume in the Colombian population according to age groups have not been established, so data from other populations with different characteristics is used in clinical practice. This increases the risk of errors in daily clinical practice and leads to equivocal behaviors, so it is important to obtain normal reference values of thyroid size in the population. The aim of this study was to estimate the reference values for normal thyroid volume in the adult population without thyroid abnormality from Pereira city.

Methods: cross-sectional observational study conducted at Clínica Comfamiliar Risaralda, with male and female population over 18 years of age without prior thyroid pathology. Ultrasound scans were performed using a General Electric ultrasound with reference Logiq P5. Descriptive statistics were used for the analysis and reference values were estimated based on age with P2.5-P97.5 centiles using regression in fractional polynomials, recommended for this type of data.

Results: the mean total volume of the thyroid gland in the population was $6.4 \pm 2.3 \mathrm{~mL}$. Regression equations in fractional polynomials were estimated for mean values and their co- 
rresponding variability in thyroid volume as a function of age. Centiles were obtained by age. Conclusions: normal thyroid size reference values were obtained in the healthy adult population from Pereira city.

Keywords: thyroid gland, ultrasonography; Colombia.

\section{Introducción}

El aumento del volumen tiroideo puede presentarse tanto en hipertiroidismo como en hipotiroidismo, siendo un hallazgo relevante durante la evaluación clínica y ecográfica de la glándula tiroides (1). Según la literatura, el tamaño tiroideo normal en la población adulta oscila entre 40-60 mm en diámetro longitudinal y entre 13-18 mm en diámetro anteroposterior (2). Según Ivanac y colaboradores, el volumen normal aproximado es 10,68 $\pm 2,83 \mathrm{~mL}$ en mujeres sanas, similar a los límites normales estimados de 10-15 mL para mujeres y 12-18 $\mathrm{mL}$ para hombres $(2,3)$.

La ecografía de tiroides es la herramienta diagnóstica más utilizada para la estimación del volumen tiroideo, la cual ha demostrado una adecuada sensibilidad y poca variación interobservador, además de su ventaja en el bajo costo con nula exposición a la radiación $(2,4,5)$. Factores relacionados, como el nivel de déficit o suplencia de yodo, edad, estatura, peso, índice de masa corporal, sexo y función tiroidea, son importantes al momento de valorar el tamaño normal en la glándula tiroides $(2,3)$.

Se han realizado varios estudios para establecer los valores de normalidad del volumen tiroideo a nivel mundial en la población pediátrica y adulta $(1,3,6-10)$. Los datos han identificado una alta variabilidad entre las poblaciones estudiadas, incluso dentro del mismo país (1). Debido a esto se recomienda establecer los valores normales locales para cada país.

En Colombia se han llevado a cabo pocos estudios de medición de volumen tiroideo en pacientes sanos, esto genera errores en la práctica clínica diaria y conlleva a conductas equivocas $(11,12)$. Este es el primer estudio que determina valores normales en adultos en nuestra región. El objetivo fue estimar los valores de referencia para el volumen tiroideo normal en la población adulta de la ciudad de Pereira sin anomalía tiroidea.

\section{Materiales y métodos}

\section{Pacientes y tipo de estudio}

Este es un estudio de corte transversal realizado entre los años 2017-2018 en la clínica Comfamiliar de la ciudad de Pereira, Colombia. Ésta es una institución de alta complejidad que atiende a parte de la población del eje cafetero (Risaralda, Quindío y Caldas) Para la inclusión de los pacientes se utilizaron los siguientes criterios: edad mayor de 18 años; personas sin patología tiroidea reconocida con niveles normales de TSH o T4 libre y que aceptaran participar en el estudio con previa firma del consentimiento informado. Se excluyeron aquellos individuos con las siguientes características: mujeres embarazadas; en uso actual de medicamentos para trastornos tiroideos o que alteren la función de la glándula como amiodarona, litio y exposición a medios de contraste iodados en los últimos 6 meses; alteración de la ecoestructura durante la realización de la ecografía y aquellos pacientes que durante el desarrollo del estudio retiraran su consentimiento de participación.

\section{Variables}

Se evaluaron variables sociodemográficas y antropométricas, como edad, sexo, talla, peso e índice de masa corporal (IMC) y variables clínicas, como el nivel de la hormona estimulante de la tiroides (TSH). Las variables ecográficas fueron volumen tiroideo total, diámetro longitudinal de lóbulos tiroideos derecho (LD-L) e izquierdo (LI-L), diámetro transverso de lóbulos tiroideos derecho (LD-T) e izquierdo (LI-T), diámetro anteroposterior de lóbulos tiroideos derecho (LD-AP) e izquierdo (LI-AP) y grosor del istmo.

\section{Recolección de la información}

A cada uno de los participantes se les realizó una evaluación clínica indagando condiciones clínicas, fisiológicas, patológicas y farmacológicas que pudieran alterar la función tiroidea y se registraron datos demográficos y antropométricos. Un endocrinólogo entrenado realizó la ecografía mediante un ecógrafo marca General Electric, referencia Logiq P5, para la toma de los datos ecográficos descritos. Los datos se almacenaron en el software de Excel, y se validaron para reducir el sesgo de información.

\section{Análisis estadísticos}

Se realizó un análisis univariado descriptivo de los datos, en el que se calculó la media y desviaciones estándar. En el orden de estimar los rangos de referencia se examinó la relación entre el volumen tiroideo y la edad con gráficos de dispersión y se procedió a seguir la metodología estadística descrita por Royston y Wright de la siguiente forma: para generar la normalidad en los datos necesaria se aplicó una transformación logarítmica a la variable de volumen tiroideo, lo que redujo la asimetría $(13,14)$. La modelación de los valores promedio y variabilidad en función de la edad se realizó mediante un modelo de regresión en polinomios fraccionales, pruebas de bondad de ajuste se aplicaron para evaluar el ajuste global de los modelos en valores promedio y de variabilidad. Curvas centiles se calcularon a partir del modelo final. Todos los cálculos se realizaron utilizando el software estadístico STATA 14.0 por medio del algoritmo sugerido por Royston (comando xriml). Asimismo se realizó un análisis de correlación con el método de Pearson entre las variables de volumen tiroideo y talla, peso e IMC. 


\section{Xultophy ${ }^{\circledR 1}$ una vez al día}

\section{Avanzando un paso más allá en el control de la diabetes tipo 2}

Con superioridad confirmada en comparación con glargina U100:

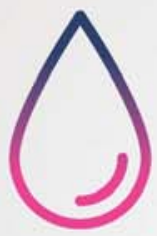

Mayor reducción

de $\mathrm{HbA}_{1 \mathrm{c}}{ }^{1,2}$

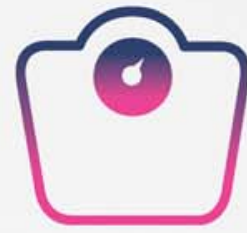

Diferencia significativa en pérdida de peso ${ }^{1,2}$

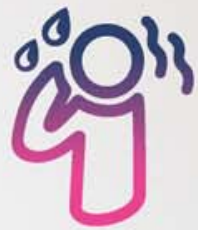

Menor tasa de hipoglucemia ${ }^{1,2}$

En pacientes con diabetes tipo 2 no controlados con glargina U100 ${ }^{1,2}$.

Un tratamiento de intensificación simple que combina insulina degludec y liraglutida en 1 dispositivo'.

\section{Xultophy}

insulina degludec/liraglutida [ADN recombinante] 
Se calculó una muestra estratificada por grupo de edad de 40 pacientes para cada uno de los 6 grupos (18-30 años, 30-40 años, 40-50 años, 60-70 años, 70-80 años y más de 80 años), con un total de 280 pacientes, suponiendo un error tipo I del 5 $\%$, una precisión del $10 \%$ y ajustado por $10 \%$ de las pérdidas de información. Como medida inicial se consideró la media y desviación estándar del volumen total de la glándula reportados por Ivanac y colaboradores (3). Este estudio contó con la aprobación del comité de ética de investigación de la clínica Comfamiliar Risaralda, en cumplimiento con la Resolución 8430 de 1993 expedida por el Ministerio de Salud de Colombia y acorde con la declaración de Helsinki.

\section{Resultados}

\section{Descripción clínica y antropométrica}

Se incluyeron 176 personas sanas sin antecedente de patología tiroidea, de las cuales 130 fueron mujeres (73,8 \%). Cabe resaltar que al inicio del estudio no se consideró una estimación separada, debido a que exigía una mayor tamaño de muestra para la estimación por los métodos utilizados por cada grupo definido y a la dificultad para conseguir voluntarios en ambos sexos; por tal motivo, la selección se dejó abierta, lo cual puede haber generado un sesgo de selección. El peso promedio en la población fue de $74 \mathrm{~kg}$ y la media de la talla fue de 1,60 metros (Tabla $\mathbf{1}$ ).

\section{Descripción ecográfica}

El promedio del diámetro anteroposterior del istmo medido fue de 2,27 mm. Respecto al lóbulo derecho se calcularon diámetros promedio en la población general de 39,1 mm x 12,1 mm x 15,9 mm (longitudinal x anteroposterior x trans- verso, respectivamente), con un promedio de volumen de lóbulo derecho de 3,6 mL. Por otro lado, el lóbulo izquierdo tuvo diámetros promedio en la población general de 38,6 mm x 10,3 mm x 14,4 mm (longitudinal x anteroposterior x transverso, respectivamente), con un promedio de volumen del lóbulo izquierdo de 2,9 mL. El promedio de volumen total en la población fue de 6,5 $\pm 2,1 \mathrm{~mL}$. En la Tabla 2 se muestran las diferentes medidas de acuerdo con cada sexo.

Tabla 1. Medidas de tendencia central de datos antropométricos

\begin{tabular}{l|c|c|c|c}
\hline & Media & DE & Mín. & Máx. \\
\hline Edad (años) & 34,6 & 11,7 & 18 & 69 \\
\hline Talla (m) & 1,6 & 0,07 & 1,43 & 1,82 \\
\hline Peso (kg) & 74 & 13,3 & 41 & 107 \\
\hline IMC (kg/m2) & 25,3 & 5,9 & 17 & 38 \\
\hline TSH (mU/L) & 2,30 & 0,7 & 0,89 & 4,49 \\
\hline
\end{tabular}

\section{Distribución de los valores normales según sexo}

Mediante modelamiento matemático descrito previamente se lograron obtener los valores de volumen total correspondientes a la edad del paciente (Figura 1). De acuerdo con esto se definió el volumen total según la edad del paciente (Anexo 1). La talla, el peso y el IMC presentaron una baja correlación con el volumen tiroideo $(r=0,26, r=0,23 \mathrm{y} \mathrm{r}=0,22$, respectivamente), por lo que la distribución del volumen tiroideo según estas variables no fue modelada.

Tabla 2. Datos ecográficos de acuerdo con el sexo

\begin{tabular}{|c|c|c|c|c|c|c|c|c|c|c|c|c|}
\hline & \multicolumn{4}{|c|}{ Femenino } & \multicolumn{4}{|c|}{ Masculino } & \multicolumn{4}{|c|}{ Total } \\
\hline & Media & $\mathrm{DE}$ & Mín. & Máx. & Media & $\mathrm{DE}$ & Mín. & Máx. & Media & $\mathrm{DE}$ & Mín. & Máx. \\
\hline Istmo-AP (mm) & 2,4 & 2,0 & 1,0 & 4,1 & 2,9 & 3,7 & 1,4 & 4,2 & 2,27 & 2,6 & 1,0 & 4,2 \\
\hline LD-L (mm) & 38,5 & 5,0 & 1,4 & 46,2 & 40,6 & 3,1 & 30,8 & 45,6 & 39,1 & 4,7 & 1,4 & 46,2 \\
\hline LD-AP (mm) & 11,8 & 3,8 & 7,2 & 16,5 & 12,9 & 2,0 & 8,0 & 16,4 & 12,1 & 3,4 & 7,2 & 16,5 \\
\hline LD-T (mm) & 15,4 & 2,7 & 2,3 & 23,6 & 17,1 & 2,5 & 12,2 & 23,2 & 15,9 & 2,7 & 2,3 & 23,6 \\
\hline LD-vol (mL) & 3,3 & 1,0 & 0,9 & 6,9 & 4,2 & 1,1 & 1,3 & 6,6 & 3,6 & 1,1 & 0,9 & 6,9 \\
\hline LI-L (mm) & 37,9 & 4,2 & 26,5 & 47,6 & 40,5 & 2,5 & 31,5 & 44,7 & 38,6 & 4,0 & 26,5 & 47,6 \\
\hline LI-AP (mm) & 9,7 & 1,9 & 0,9 & 17,3 & 12,2 & 2,4 & 8 & 19,2 & 10,3 & 2,4 & 0,91 & 19,2 \\
\hline LI-T (mm) & 14,1 & 2,8 & 1,5 & 21,3 & 15,1 & 2,2 & 11,2 & 20,1 & 14,4 & 2,7 & 1,5 & 21,3 \\
\hline LI-vol (mL) & 2,7 & 1,4 & 0,9 & 14,4 & 3,6 & 0,8 & 2,3 & 5,8 & 2,9 & 1,3 & 0,9 & 14,4 \\
\hline $\begin{array}{l}\text { Volumen } \\
\text { tiroideo total }(\mathrm{mL})\end{array}$ & 6,0 & 2,0 & 2,4 & 17,3 & 7,9 & 1,7 & 4,0 & 11,4 & 6,5 & 2,1 & 2,4 & 17,3 \\
\hline
\end{tabular}


Figura 1. Volumen tiroideo según centiles por edad.

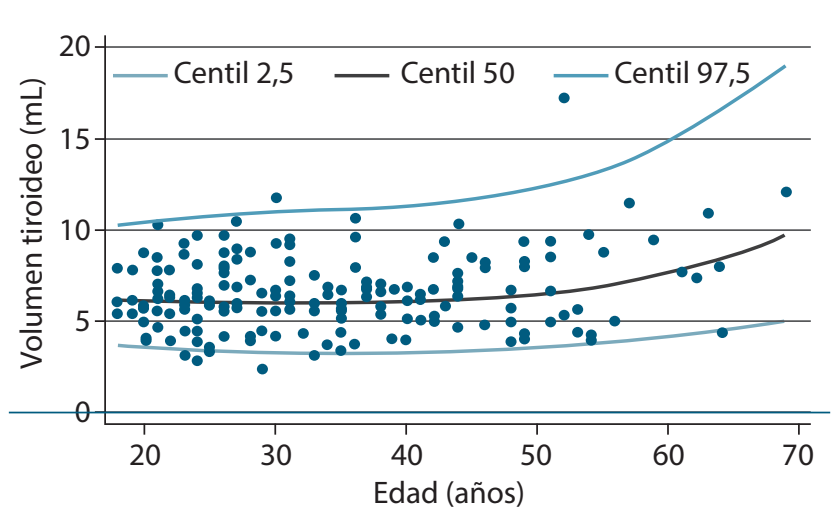

\section{Discusión}

Este es el primer estudio en nuestra región que establece los valores de normalidad del volumen tiroideo en personas adultas y sin patología tiroidea. Este estudio ha incluido datos de normalidad de pacientes con diferentes edades, hasta los 70 años, en el que se identificó una tendencia estable hasta los 50 años, a partir de la cual, el volumen presenta un incremento con la edad. Determinar el volumen tiroideo es de relevancia en la práctica clínica para el diagnóstico y tratamiento de patologías tiroideas y deficiencia de iodo, entre ellas, bocio multinodular, cáncer de tiroides, hiper e hipotiroidismo, tiroiditis, entre otras. Además de evaluar la eficacia de la terapia con levotiroxina e indicación de cirugía mínima invasiva (15).

Múltiples estudios en los que se establecen los valores de normalidad del volumen tiroideo se han llevado a cabo alrededor del mundo (2, 6-10). Estos estudios han identificado una alta variabilidad entre diferentes países e incluso dentro del mismo país debido a diferencias étnicas, de suplencia de iodo, edad, sexo, talla, patología tiroidea, entre otras. El estudio de Dvoráková y colaboradores demostró, en una población con suplencia adecuada de iodo, que hubo un incremento relacionado con la edad en ambos sexos (F-ratio = 1,99, $p<0,0001$ ), con una meseta moderada en las mujeres desde los 30 hasta los 55 años, a partir del cual se encontró un incremento rápido en ambos sexos (8). Estos resultados son similares a nuestro estudio, compuesto principalmente por el sexo femenino (74\%), y al estudio de Aydıner y colaboradores ( $\mathrm{r}=0,306 ; p$ $<0,0001$ ) cuando analizaron a los mayores de 18 años (1).

En cuanto al volumen tiroideo, nuestro estudio identificó una media de 6,5 $\pm 2,1 \mathrm{~mL}$ (rango 2,4-17,3 mL), siendo mayor en el sexo masculino (media 7,9 $\pm 1,7 \mathrm{~mL}$ ) que en el femenino (media: 6,0 $\pm 2,0 \mathrm{~mL}$ ), hallazgo que es menor que el reportado en el estudio de Ivanac y colaboradores realizado en la población femenina (media 10,68 \pm 2,83 mL (rango 5,71-17,09 mL) y menor al de Sahin y colaboradores realizado en población adulta de ambos sexos (media 12,98 $\pm 2,53 \mathrm{~mL}$ ), con mayor volumen $(p<0,05)$ identificado en el sexo masculino (3).
Nuestros hallazgos en cuanto a volumen tiroideo fueron similares a los de Mohamed y colaboradores en Sudan, donde evaluaron a 103 pacientes de ambos sexos y adultos con una media de 6,44 $\pm 2,44 \mathrm{~mL}$ (siendo mayor en hombres) (10). Asimismo, el volumen promedio fue similar en las mujeres del estudio de Gómez y colaboradores en España, con una media de 6,19 mL (intervalo de confianza 6,02-6,92) en adultos sanos, sin deficiencia de iodo, y al estudio de Turcios y colaboradores en Cuba, con un promedio de 6,6 $\pm 0,26 \mathrm{~mL}(16,17)$.

Es importante destacar que múltiples estudios han identificado una correlación importante del volumen tiroideo con parámetros antropométricos, como la talla, superficie, IMC y peso $(1,3,16,18-22)$; sin embargo, en nuestro estudio la correlacion entre talla, peso e IMC frente al volumen tirodeo fue baja. En Colombia se han realizado pocos estudios sin incluir población adulta sana para establecer valores normales, todos llevados a cabo en la ciudad de Bogotá $(11,12)$. El estudio de Schmulson y colaboradores incluyó 10 pacientes con bocio clínico para evaluar la concordancia con 10 individuos normales, como parte de un protocolo para determinar la respuesta del bocio simple a la terapia supresiva (12). Asimismo, el estudio de Gonzales y colaboradores evaluó a 591 niños y se establecieron sus volúmenes tiroideos por rangos de edad (11). Estos autores identificaron valores menores de los establecidos por la Organización Mundial de la Salud y una alta correlación con la superficie corporal.

Dentro de las limitaciones de este estudio destacamos la limitación estadística al no lograr el total del tamaño de muestra calculado que podría influir sobre las estimaciones realizadas en el valor promedio y sus centiles del volumen tiroideo, sin embargo, la premisa en el análisis fue modelar el comportamiento del valor en un rango de edad sin realizar estimaciones de confiabilidad (por ejemplo, intervalos de confianza) sobre las mismas. Como fortalezas resaltamos que se realizó un análisis estadístico sólido y confiable. Este estudio implica datos de referencia de volumen tiroideo para la población local y un estándar para la población adulta nacional.

\section{Conclusiones}

Los valores de referencia normales de volumen tiroideo en la población adulta sana de la ciudad de Pereira, Colombia corresponden a una media de 6,5 $\pm 2,1 \mathrm{~mL}$, siendo mayor en el sexo masculino que en el femenino y con un evidencia de incremento a partir de los 50 años.

\section{Conflictos de interés}

Ninguno por declarar.

\section{Fuente de financiación}

Propia de los autores. 
- La educación del paciente es parte primordial del tratamiento y es la base para que logre sus metas. ${ }^{(1)}$

- La presencia de Lipohipertrofia, reduce la absorción de insulina. La detección y manejo reducen los costos del tratamiento $^{(1)}$

- Dispositivos de calidad y un buen programa educativo son efectivos en pacientes insulinorrequirientes ${ }^{(2)}$

\section{Programa Educativo para paciente con diabetes de BD Colombia}

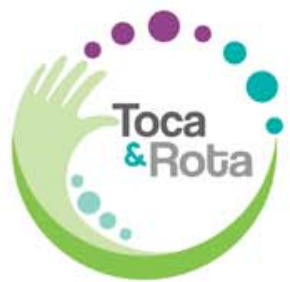

Que la diabetes no te marque, se parte y comparte.

\section{BD Ultra-Fine ${ }^{\circledR}$ Nano Easy Flowhology $_{\text {The }}^{\text {math }}$}

Aguja para Pen de insulina con pared extradelgada que ofrece mayor diámetro interno aumentando la rapidez de flujo

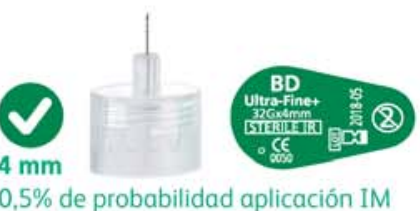

BD Ultra-fine'

Nano Penta Point " EasyFlow" Aguja para PEN de insulina

Caja $\times 100$ Unidades $4 \mathrm{~mm}$ - 326 Ref: 320489

INVIMA2018DM-0017818
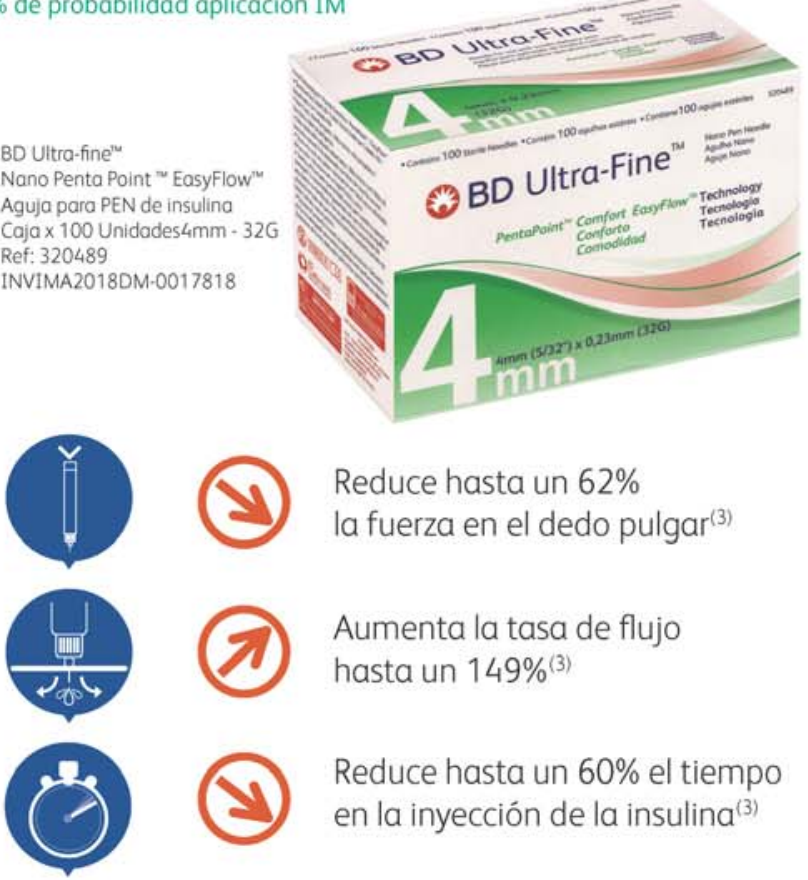

(1)

Reduce hasta un $62 \%$

la fuerza en el dedo pulgar ${ }^{(3)}$

2. Aumenta la tasa de flujo

hasta un $149 \%^{(3)}$

Reduce hasta un $60 \%$ el tiempo en la inyección de la insulina ${ }^{(3)}$

M. Blance ef ol. / Drabetes \& Metabolism 39 (2013) 445-453

Lipohipertrofic conocimiento y educación al tratamiento con insulina en la diabetes melitus. Sandra Milena Cano Ibarm. Gloria Lastre Amell, María Alejandra Oróstegui Santander Revista Med 2018.26

on preference. confidence and Berube 1, Kasler Taub K, Hirsch L Insulin pen needles: effects of ext 


\section{Referencias}

1. Aydıner Ö, Karakoç Aydıner E, Akpınar İ, Turan S, Bereket A. Normative data of thyroid volume-ultrasonographic evaluation of 422 subjects aged 0-55 years. J Clin Res Pediatr Endocrinol. 2015;7(2):98-101.

2. Chaudhary V, Bano S. Thyroid ultrasound. Indian J Endocrinol Metab. 2013;17(2):219-27

3. Ivanac G, Rozman B, Skreb F, Brkljacic B, Pavic L. Ultrasonographic measurement of the thyroid volume. Coll Antropol. 2004;28(1):287-91.

4. Pleśniak J, Urbański S. Comparative thyroid gland volume by two methods: ultrasonography and planar scintigraphy. Pol J Radiol. 2012;77(2):19-21.

5. Nygaard B, Nygaard T, Court-Payen M, Jensen LI, Soe-Jensen P, Gerhard Nielsen K, et al. Thyroid volume measured by ultrasonography and CT. Acta Radiol. 2002;43(3):269-74.

6. Szybinski Z, Trofimiuk-Muldner M, Buziak-Bereza M, Walczycka L, Hubalewska-Dydejczyk A. Reference values for thyroid volume established by ultrasound in Polish schoolchildren. Endokrynol Pol. 2012;63(2):104-9.

7. Zhou J, Huang X, Zhu W, Qin G. Study on the upper limit and its revision method of normal thyroid volume of children of 8-10 years-old in Zhejiang Province [abstract]. Wei Sheng Yan Jiu. 2007;36(4):517-9.

8. Dvorakova M, Bilek R, Cerovska J, Hill M, Novak Z, Vavrejnova V, et al. The volumes of the thyroid gland in adults aged 18-65 years in the Czech Republic--determination of the norms [abstract]. Vnitr Lek. 2006;52(1):57-63.

9. Sahin E, Elboga U, Kalender E. Regional reference values of thyroid gland volume in Turkish adults. Srp Arh Celok Lek. 2015;143(3-4):141-5.

10. Yousef M, Sulieman A, Ahmed B, Abdella A, Eltom K. Local reference ranges of thyroid volume in Sudanese normal subjects using ultrasound. J Thyroid Res. 2011;2011:935141.

11. González M, González CP, Sanabria Á. Determinación ecográfica del volumen normal de la glándula tiroides en una población pediátrica de Bogotá, Colombia. Biomédica. 2006;26:95-100.

12. Schmulson MJ, Palacio A, Dennis R, Jiménez L. Evaluación de la ecografia en la medición del volumen tiroideo. Acta Med Colomb. 1992;17(6):427-31.
13. Royston P, Wright EM. A method for estimating age-specific reference intervals ('normal ranges') based on fractional polynomials and exponentia transformation. J R Stat Soc Ser A Stat Soc. 1998;161(1):79-101.

14. Wright EM, Royston P. Simplified estimation of age-specific reference intervals for skewed data. Stat Med. 1997;16(24):2785-803.

15. Grussendorf M, Reiners C, Paschke R, Wegscheider K. Reduction of thyroid nodule volume by levothyroxine and iodine alone and in combination: a randomized, placebo-controlled trial. J Clin Endocrinol Metab. 2011;96(9):2786-95.

16. Gomez JM, Maravall FJ, Gomez N, Guma A, Soler J. Determinants of thyroid volume as measured by ultrasonography in healthy adults randomly selected. Clin Endocrinol (Oxf). 2000;53(5):629-34.

17. Turcios S, Lence-Anta JJ, Santana JL, Pereda CM, Velasco M, Chappe M, et al. Thyroid volume and its relation to anthropometric measures in a healthy cuban population. Eur Thyroid J. 2015;4(1):55-61.

18. Hegedus L, Perrild H, Poulsen LR, Andersen JR, Holm B, Schnohr P, et al The determination of thyroid volume by ultrasound and its relationship to body weight, age, and sex in normal subjects. J Clin Endocrinol Metab. 1983;56(2):260-3.

19. Jafary F, Aminorroaya A, Amini M, Adibi A, Sirous M, Roohi E, et al. Thyroid incidentaloma in Isfahan, Iran - A population-based study. Endokrynol Pol. 2008;59(4):316-20.

20. Barrere X, Valeix P, Preziosi P, Bensimon M, Pelletier B, Galan P, et al. De terminants of thyroid volume in healthy French adults participating in the SU.VI.MAX cohort. Clin Endocrinol (Oxf). 2000;52(3):273-8.

21. Eray E, Sari F, Ozdem S, Sari R. Relationship between thyroid volume and iodine, leptin, and adiponectin in obese women before and after weight loss. Med Princ Pract. 2011;20(1):43-6.

22. Veres C, Garsi JP, Rubino C, Pouzoulet F, Bidault F, Chavaudra J, et al. Thyroid volume measurement in external beam radiotherapy patients using CT imaging: correlation with clinical and anthropometric characteristics. Phys Med Biol. 2010;55(21):N507-19. 


\section{ANEXOS}

Anexo 1. Volumen tiroideo total (mL) en centiles según la edad de una población adulta de la ciudad de Pereira, Colombia

\begin{tabular}{|c|c|c|c|c|c|c|c|}
\hline Edad & P 2,5 & P 50 & P 97,5 & Edad & P 2,5 & P 50 & P 97,5 \\
\hline 18 & 3,61 & 6,07 & 10,23 & 40 & 3,23 & 6,05 & 11,34 \\
\hline 19 & 3,55 & 6,06 & 10,35 & 41 & 3,23 & 6,07 & 11,41 \\
\hline 20 & 3,51 & 6,05 & 10,45 & 42 & 3,24 & 6,10 & 11,48 \\
\hline 21 & 3,47 & 6,04 & 10,53 & 43 & 3,25 & 6,13 & 11,55 \\
\hline 22 & 3,43 & 6,03 & 10,60 & 44 & 3,27 & 6,16 & 11,63 \\
\hline 23 & 3,40 & 6,02 & 10,66 & 45 & 3,28 & 6,20 & 11,72 \\
\hline 24 & 3,38 & 6,01 & 10,72 & 46 & 3,30 & 6,24 & 11,82 \\
\hline 25 & 3,35 & 6,01 & 10,76 & 48 & 3,34 & 6,34 & 12,05 \\
\hline 26 & 3,33 & 6,00 & 10,80 & 49 & 3,37 & 6,40 & 12,18 \\
\hline 27 & 3,31 & 5,99 & 10,84 & 51 & 3,43 & 6,54 & 12,47 \\
\hline 28 & 3,29 & 5,99 & 10,88 & 52 & 3,46 & 6,62 & 12,64 \\
\hline 29 & 3,28 & 5,98 & 10,91 & 53 & 3,50 & 6,70 & 12,82 \\
\hline 30 & 3,27 & 5,98 & 10,93 & 54 & 3,54 & 6,79 & 13,02 \\
\hline 31 & 3,25 & 5,98 & 10,97 & 55 & 3,59 & 6,89 & 13,23 \\
\hline 32 & 3,24 & 5,98 & 11,00 & 56 & 3,64 & 7,00 & 13,47 \\
\hline 33 & 3,24 & 5,98 & 11,04 & 57 & 3,70 & 7,12 & 13,72 \\
\hline 34 & 3,23 & 5,98 & 11,07 & 59 & 3,83 & 7,39 & 14,28 \\
\hline 35 & 3,23 & 5,99 & 11,11 & 61 & 3,98 & 7,71 & 14,94 \\
\hline 36 & 3,22 & 5,99 & 11,15 & 62 & 4,06 & 7,89 & 15,32 \\
\hline 37 & 3,22 & 6,00 & 11,19 & 63 & 4,16 & 8,08 & 15,72 \\
\hline 38 & 3,22 & 6,02 & 11,24 & 64 & 4,26 & 8,29 & 16,16 \\
\hline 39 & 3,22 & 6,03 & 11,29 & 69 & 4,90 & 9,64 & 18,95 \\
\hline
\end{tabular}

